\title{
Dynamics of a bilayer membrane with membrane-solvent partial slip boundary conditions
}

\author{
Kento Yasuda and Shigeyuki Komura* \\ Department of Chemistry, Graduate School of Science and Engineering, \\ Tokyo Metropolitan University, Tokyo 192-0397, Japan \\ Ryuichi Okamoto \\ Research Institute for Interdisciplinary Science, Okayama University, Okayama 700-8530, Japan \\ Jean-Baptiste Fournie话 \\ Université Paris Diderot, Sorbonne Paris Cité, Laboratoire Matière et \\ Systèmes Complexes (MSC), UMR 7057 CNRS, F-75205 Paris, France
}

(Dated: November 9, 2018)

\begin{abstract}
We discuss the dynamics of a bilayer membrane with partial slip boundary conditions between the monolayers and the bulk fluid. Using Onsager's variational principle to account for the associated dissipations, we derive the coupled dynamic equations for the membrane height and the excess lipid density. The newly introduced friction coefficients appear in the renormalized fluid viscosities. For ordinary lipid bilayer membranes, we find that it is generally justified to ignore the effects of permeation and parallel slip at the membrane surface.
\end{abstract}

Much attention has been paid to artificial lipid bilayer membranes as model systems of biological cell membranes [1]. The fluidity of biomembranes is guaranteed mainly by the lipid molecules, which are in the liquid crystalline state at physiological temperatures. Biomembranes exhibit a wide variety of complex phenomena, in both statics and dynamics, since lipid densities, membrane deformation and surrounding fluids are coupled to each other. In early theoretically studies, the relaxation rate of a lipid membrane was discussed by regarding it as a tensionless elastic sheet undergoing out-of-plane fluctuations [2]. The membrane relaxation was then shown to be dominated by the bending rigidity and the viscosity of the surrounding bulk fluid.

Later on, Merkel et al. [3] and Seifert and Langer [4] considered both the inter-monolayer friction and the twodimensional (2D) hydrodynamics of each monolayer. Importantly, they obtained another relaxation mode which is associated with the density difference between the two monolayers and is further coupled to the bending mode. Such a relaxation of the density fluctuation is dominated by the inter-monolayer friction. The existence of the predicted compressional mode has been confirmed in the recent experiment [5]. After the work by Seifert and Langer, the bilayer nature of lipid membranes has been explicitly considered for two-component bilayer membranes [6] and spherically closed bilayer vesicles 7 [ 9]. More recently, the present authors have discussed the dynamics of a bilayer membrane coupled to a $2 \mathrm{D}$ cytoskeleton [10].

In all of these works, a partial slip boundary condition between the two monolayers has been employed [4].

\footnotetext{
* komura@tmu.ac.jp

$\dagger$ jean-baptiste.fournier@univ-paris-diderot.fr
}

Conversely, the associated models have always assumed a no-slip boundary condition between the monolayer and the outer bulk fluid (solvent). Precisely speaking, this noslip boundary condition requires that the velocity of the bulk fluid has the following properties at the monolayer surfaces: (i) the velocity component normal to the membrane coincides with the change rate of the out-of-plane membrane displacement, so that the bulk fluid cannot permeate through the membrane, and (ii) the lateral velocity component coincides with the fluid velocity of the monolayer.

In this paper, we discuss the dynamics of a bilayer membrane with partial slip boundary conditions between the monolayers and the bulk fluid, i.e., we study the case when the above conditions (i) and (ii) are violated. It is of importance to know which of the slipping modes (intermonolayer or monolayer-solvent) dominates the membrane dynamics at large-wavenumber excitations. We use the framework of Onsager's variational principle 11] to obtain the governing hydrodynamic equations 12 . In particular, the friction between the monolayer and the bulk fluid is taken into account through newly introduced dissipation functions. In order to highlight the effects of the monolayer-solvent partial slip boundary condition, we shall closely follow the notations in Ref. [12].

As shown in Fig. 1, we describe the membrane shape by a height function $h(\mathbf{r}, t)$, where the $2 \mathrm{D}$ vector $\mathbf{r}=(x, y)$ is a generic point in a reference plane. We use $\rho^{+}(\mathbf{r}, t)$ and $\rho^{-}(\mathbf{r}, t)$ to describe relative excess mass densities of the upper and the lower monolayers, respectively. The two lipid monolayers are regarded as $2 \mathrm{D}$ fluid sheets with a shear viscosity $\eta_{2}$ and a dilatational viscosity $\lambda_{2}$. The lateral velocity of each monolayer is denoted $v_{i}^{ \pm}(\mathbf{r}, t)$, with $i \in\{x, y\}$. The outer bulk fluids are assumed identical on both sides of the membrane and are characterized by the bulk fluid viscosity $\eta$, the pressure $P^{ \pm}(\mathbf{R}, t)$, and the 


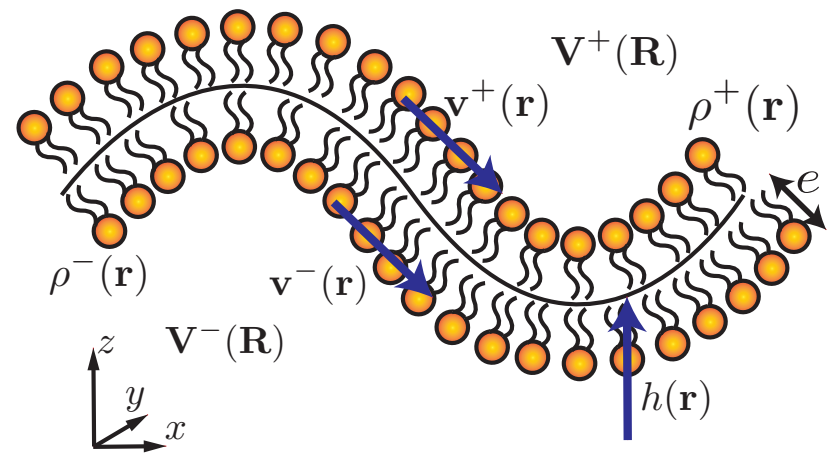

FIG. 1. Schematic picture of a laterally compressible bilayer membrane. The deformation of the membrane is described by the height variable $h(\mathbf{r})$ and the excess lipid mass densities of the upper and the lower monolayers by $\rho^{ \pm}(\mathbf{r})$. The lateral velocities of the upper monolayer and the lower monolayer are $\mathbf{v}^{ \pm}(\mathbf{r}, t)$. The velocities of the bulk fluid in the upper and the lower side of the membrane are $\mathbf{V}^{ \pm}(\mathbf{R}, t)$.

velocity $V_{\alpha}^{ \pm}(\mathbf{R}, t)$, where $\alpha \in\{x, y, z\}$ and $\mathbf{R}=(\mathbf{r}, z)$ is a three-dimensional vector.

The dynamical equations can be obtained without any ambiguity from the extremalization of the Rayleighian [11] of the whole system, i.e., the bilayer membrane and the bulk fluid. In general, the Rayleighian consists of a dissipation function plus the time derivative of a free energy. The conservation laws and the boundary conditions are taken into account by introducing Lagrange multipliers. Regarding the bulk solvent as an incompressible fluid, we require $\partial_{\alpha} V_{\alpha}^{ \pm}=0$, whereas the mass conservation is expressed by $\dot{\rho}^{ \pm}+\partial_{i} v_{i}^{ \pm}=0$. In this paper, we shall later introduce new friction coefficients between the monolayers and the bulk fluid.

Concerning the boundary condition of the normal component of the velocities, we require that the normal velocities of the upper and lower monolayers coincide. However, since we shall use partial slip boundary conditions between the monolayers and the bulk fluid, the velocities of the membrane and the bulk fluid should be different in general. Hence we only require the following boundary conditions regarding the bulk fluid velocities:

$$
\left.V_{z}^{+}\right|_{z=0}=\left.V_{z}^{-}\right|_{z=0},
$$

which ensures that no water is stocked by the membrane.

Next, we consider the dissipation functions of the system. The dissipation functions corresponding to the bulk fluid $\mathcal{P}_{b}^{ \pm}$and the $2 \mathrm{D}$ fluid monolayers $\mathcal{P}_{s}^{ \pm}$are given by

$$
\mathcal{P}_{b}^{ \pm}=\int d^{3} R \eta D_{\alpha \beta}^{ \pm} D_{\alpha \beta}^{ \pm}
$$

where $D_{\alpha \beta}^{ \pm}=\left(\partial_{\alpha} V_{\beta}^{ \pm}+\partial_{\beta} V_{\alpha}^{ \pm}\right) / 2$, and

$$
\mathcal{P}_{s}^{ \pm}=\int d^{2} r\left(\eta_{2} d_{i j}^{ \pm} d_{i j}^{ \pm}+\frac{\lambda_{2}}{2} d_{i i}^{ \pm} d_{j j}^{ \pm}\right)
$$

where $d_{i j}^{ \pm}=\left(\partial_{i} v_{j}^{ \pm}+\partial_{j} v_{i}^{ \pm}\right) / 2$. Furthermore, the dissipation due to the inter-monolayer friction is given by

$$
\mathcal{P}_{i}=\int d^{2} r \frac{b}{2}\left(v_{i}^{+}-v_{i}^{-}\right)^{2},
$$

in which the friction coefficient $b$ between the two monolayers appears.

In contrast to Ref. [12], we shall consider here the dissipation which occurs at the boundaries between the monolayers and the bulk fluid. The friction in the $x, y$ direction is proportional to the relative velocity $v_{i}^{ \pm}-$ $\left.V_{i}^{ \pm}\right|_{z=0}$, whereas that in the $z$-direction is determined by $\dot{h}-\left.V_{z}^{+}\right|_{z=0}$, where $\dot{h}$ indicates the time derivative of $h$. Hence the corresponding dissipation functions can be written as

$$
\begin{aligned}
& \mathcal{P}_{\|}^{ \pm}=\int d^{2} r \frac{b_{\|}}{2}\left(v_{i}^{ \pm}-\left.V_{i}^{ \pm}\right|_{z=0}\right)^{2}, \\
& \mathcal{P}_{\perp}=\int d^{2} r \frac{b_{\perp}}{2}\left(\dot{h}-\left.V_{z}^{+}\right|_{z=0}\right)^{2},
\end{aligned}
$$

where $b_{\|}$is the friction coefficient in the parallel direction, and $b_{\perp}$ is that in the normal direction. These are the new dissipation functions that we consider in this paper. Notice that the inverse of $b_{\perp}$ is known as the membrane permeation coefficient [13].

Next we briefly discuss the free energy of a laterally compressible bilayer membrane with finite thickness. The elasticity of a flexible membrane is generally characterized by a surface tension $\sigma$ and a bending rigidity $\kappa$. Using these quantities, the free energy per unit area of a membrane is given by $\frac{1}{2} \sigma(\nabla h)^{2}+\frac{1}{2} \kappa c^{2}$ where $c \approx \nabla^{2} h$ is twice the mean curvature. We also consider the coupling between the membrane curvature and the lipid density in the monolayers [4]. When the membrane has a positive mean curvature, the upper monolayer is stretched while the lower one is compressed. The amount of stretching or compression is simply given by $e c$, where $e$ is the distance between the monolayer neutral surface and the membrane mid-surface (see Fig. 11). Introducing the monolayer stretching coefficient $k$, one can write down the total free energy as

$$
\begin{aligned}
\mathcal{H}= & \int d^{2} r\left[\frac{\sigma}{2}(\nabla h)^{2}+\frac{\kappa}{2}\left(\nabla^{2} h\right)^{2}\right. \\
& \left.+\frac{k}{2}\left(\rho^{+}+e \nabla^{2} h\right)^{2}+\frac{k}{2}\left(\rho^{-}-e \nabla^{2} h\right)^{2}\right] .
\end{aligned}
$$

The whole set of dynamical equations can be obtained by extremalizing the Rayleighian with respect to all of the dynamical variables [11]. The Rayleighian is given by the sum of all the dissipation functions and the time derivative of the free energy, i.e., $\mathcal{R}=\mathcal{P}_{b}^{+}+\mathcal{P}_{b}^{-}+\mathcal{P}_{s}^{+}+$ $\mathcal{P}_{s}^{-}+\mathcal{P}_{i}+\mathcal{P}_{\|}^{+}+\mathcal{P}_{\|}^{-}+\mathcal{P}_{\perp}+\dot{\mathcal{H}}$. The incompressibility condition and the mass conservation condition, as shown before, are taken into account by the method of Lagrange multiplier. Extremalizing $\mathcal{R}$ with respect to the fields $v^{ \pm}$, $V^{ \pm}, \dot{h}, \dot{\rho}^{ \pm}$and the Lagrange multiplier fields, we finally 
obtain the dynamical equations for a compressible bilayer membrane with partial slip boundary conditions between the monolayers and the bulk fluids.

The equations for the outer bulk fluid are the Stokes equation and the incompressibility condition which are given by $-\eta \nabla^{2} V_{\alpha}^{ \pm}+\partial_{\alpha} P^{ \pm}=0$ and $\partial_{\alpha} V_{\alpha}^{ \pm}=0$, respectively. Solving these equations with the use of a $2 \mathrm{D}$ Fourier transform of the variables such as

$$
X(\mathbf{q}, z)=\int d^{2} r X(\mathbf{R}) e^{-i \mathbf{q} \cdot \mathbf{r}},
$$

we obtain the bulk fluid velocity as

$$
\begin{aligned}
& V_{z}^{ \pm}(\mathbf{q}, z)=\left(A^{ \pm}+B^{ \pm} z\right) e^{\mp q z}, \\
& V_{\|}^{ \pm}(\mathbf{q}, z)=i\left(B^{ \pm} / q \mp A^{ \pm} \mp B^{ \pm} z\right) e^{\mp q z},
\end{aligned}
$$

where $V_{\|}^{ \pm}(\mathbf{q}, z)$ is the component of the bulk fluid velocity parallel to the direction of $\mathbf{q}$, while $A^{ \pm}$and $B^{ \pm}$are coefficients yet to be determined. In the above, we have assumed that $V_{\alpha}^{ \pm} \rightarrow 0$ for $z \rightarrow \pm \infty$. In addition, the pressure is given by $P^{ \pm}(\mathbf{q}, z)=2 \eta B^{ \pm} e^{\mp q z}$. Notice that the component of the bulk fluid velocity perpendicular to $\mathbf{q}$, denoted by $V_{\perp}^{ \pm}(\mathbf{q}, z)$, is completely decoupled from the other components.

The boundary conditions at the membrane, $z=0$, are Eq. (1) and

$$
\begin{aligned}
& \mp \eta\left(\partial_{z} V_{i}^{ \pm}+\partial_{i} V_{z}^{ \pm}\right)-b_{\|}\left(v_{i}^{ \pm}-V_{i}^{ \pm}\right)=0 \\
& -2 \eta\left(\partial_{z} V_{z}^{+}-\partial_{z} V_{z}^{-}\right)+P^{+}-P^{-}-b_{\perp}\left(\dot{h}-V_{z}^{+}\right)=0
\end{aligned}
$$

which are the force balance equations in the perpendicular and parallel directions to the membrane, respectively, in the presence of partial slip effects. These boundary conditions are now used to obtain the unknown coefficients as

$$
\begin{aligned}
A^{ \pm} & =\frac{b_{\perp}}{b_{\perp}+4 \eta q} \dot{h}(\mathbf{q}), \\
B^{ \pm} & = \pm q \frac{b_{\perp}}{b_{\perp}+4 \eta q} \dot{h}(\mathbf{q})-i q \frac{b_{\|}}{b_{\|}+2 \eta q} v_{\|}^{ \pm}(\mathbf{q}) .
\end{aligned}
$$

Note that $v_{\|}^{ \pm}(\mathbf{q})$ in Eq. (14) is the component of the membrane velocity parallel to $\mathbf{q}$.

The force balance equations for the membrane itself are given by

$$
\begin{aligned}
\tilde{\kappa} \nabla^{4} h & -\sigma \nabla^{2} h+k e \nabla^{2}\left(\rho^{+}-\rho^{-}\right)+P^{+}-2 \eta \partial_{z} V_{z}^{+} \\
& -P^{-}+2 \eta \partial_{z} V_{z}^{-}=0
\end{aligned}
$$

and

$$
\begin{aligned}
-\eta_{2} \nabla^{2} v_{i}^{ \pm} & -\left(\eta_{2}+\lambda_{2}\right) \partial_{i} \nabla \cdot v^{ \pm}+b\left(v_{i}^{ \pm}-v_{i}^{\mp}\right) \\
& +k \partial_{i}\left(\rho^{ \pm} \pm e \nabla^{2} h\right) \mp \eta\left(\partial_{z} V_{i}^{ \pm}+\partial_{i} V_{z}^{ \pm}\right)=0 .
\end{aligned}
$$

Using the solutions for the bulk fluid as calculated above, we finally obtain the dynamical equations for the membrane height $h$ and the density difference $\rho=\rho^{+}-\rho^{-}$ as

$$
\left(\begin{array}{c}
\dot{h}(\mathbf{q}, t) \\
\dot{\rho}(\mathbf{q}, t)
\end{array}\right)=-M(q)\left(\begin{array}{c}
h(\mathbf{q}, t) \\
\rho(\mathbf{q}, t)
\end{array}\right)
$$

where

$$
M(q)=\left(\begin{array}{cc}
\frac{\sigma q+\tilde{\kappa} q^{3}}{4 \eta_{\perp}(q)} & -\frac{k e q}{4 \eta_{\perp}(q)} \\
-\frac{k e q^{4}}{b+\eta_{\|}(q) q+\eta_{s} q^{2}} & \frac{k q^{2}}{2\left[b+\eta_{\|}(q) q+\eta_{s} q^{2}\right]}
\end{array}\right)
$$

In the above matrix, we have defined the effective bending rigidity and the effective surface viscosity as

$$
\tilde{\kappa}=\kappa+2 k e^{2}, \quad \eta_{s}=\eta_{2}+\frac{\lambda_{2}}{2},
$$

whereas the wavenumber dependent renormalized viscosities are

$$
\eta_{\perp}(q)=\frac{\eta b_{\perp}}{b_{\perp}+4 \eta q}, \quad \eta_{\|}(q)=\frac{\eta b_{\|}}{b_{\|}+2 \eta q} .
$$

(In Ref. 4], the density difference was defined as $\rho=$ $\left(\rho^{+}-\rho^{-}\right) / 2$. This leads to a somewhat different appearance of the corresponding relaxation matrix.) We do not discuss here the dynamics of the density sum $\bar{\rho}=\rho^{+}+\rho^{-}$since it is completely decoupled from the other variables [4]. First we note that our result generalizes that of Seifert and Langer [4], because the bulk viscosity $\eta$ is now replaced either by $\eta_{\perp}(q)$ or $\eta_{\|}(q)$. In other words, both $\eta_{\perp}$ and $\eta_{\|}$reduce to $\eta$ when $b_{\perp} \gg 4 \eta q$ and $b_{\|} \gg 2 \eta q$, respectively, corresponding to the previous no-slip boundary condition. Equations (17) and (18) are the main result of this paper.

Next we discuss the wavenumber dependence of the relaxation rates, which are the two eigenvalues of the matrix in Eq. (18). First of all, one can demonstrate that the two eigenvalues are real and positive in all situations. This property is ensured by the positivity of all the dissipation functions as well as the positivity of the static parameters such as $\kappa$ and $k$. In order to see clearly the effects of the partial slip boundary conditions, we consider here the tensionless case and set $\sigma=0$. The asymptotic analysis of the relaxation matrix yields the following wavenumber dependences of the two eigenvalues:

$$
\gamma_{1} \approx \begin{cases}(k / 2 b) q^{2}, & q \ll q_{1}, \\ (\tilde{\kappa} / 4 \eta) q^{3}, & q_{1} \ll q \ll q_{2}, \\ \left(\tilde{\kappa} / b_{\perp}\right) q^{4}, & q_{2} \ll q,\end{cases}
$$

$$
\gamma_{2} \approx \begin{cases}(\kappa / 4 \eta) q^{3}, & q \ll q_{1}, \\ (k / 2 b)(\kappa / \tilde{\kappa}) q^{2}, & q_{1} \ll q \ll q_{3}, \\ \left(k / 2 \eta_{s}\right)(\kappa / \tilde{\kappa}), & q_{3} \ll q .\end{cases}
$$




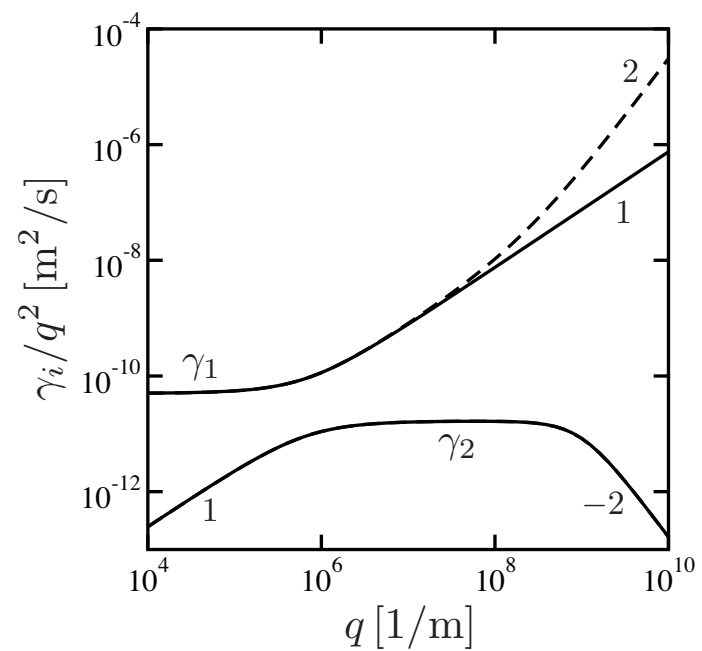

FIG. 2. The relaxation rates $\gamma_{1}$ and $\gamma_{2}$ as a function of the wavenumber $q$. Both are normalized by $q^{2}$. The parameter values, given in the text, are those of a tensionless ordinary lipid membrane. The solid and dashed lines correspond to $b_{\perp}=10^{12} \mathrm{~J} \cdot \mathrm{s} / \mathrm{m}^{4}$ (ordinary membrane) and $b_{\perp}=10^{6} \mathrm{~J} \cdot \mathrm{s} / \mathrm{m}^{4}$ (very permeable membrane), respectively. The different numbers indicate the slope representing the exponent of the power-law behaviors.

Here the three crossover wavenumbers are $q_{1}=2 \eta k /(b \tilde{\kappa})$, $q_{2}=b_{\perp} /(4 \eta)$, and $q_{3}=\sqrt{b / \eta_{s}}$. Among these, $q_{1}$ and $q_{3}$ were discussed before [4], while $q_{2}$ introduces a new length scale.

The parameters of ordinary lipid membranes have the following order of magnitudes: $e \approx 10^{-9} \mathrm{~m}, k \approx$ $0.1 \mathrm{~J} / \mathrm{m}^{2}, \kappa \approx 10^{-19} \mathrm{~J}, b \approx 10^{9} \mathrm{~J} \cdot \mathrm{s} / \mathrm{m}^{4}, \eta_{s} \approx$ $10^{-9} \mathrm{~J} \cdot \mathrm{s} / \mathrm{m}^{2}$, and $\eta \approx 10^{-3} \mathrm{~J} \cdot \mathrm{s} / \mathrm{m}^{3}$ (pure water) [12]. Note that these values are consistent with the relation $b \approx \eta_{s} / e^{2}$. Likewise, it seems reasonable to estimate the friction coefficient $b_{\|}$between the monolayer and the bulk fluid by using the water viscosity $\eta$ and the water molecular size $a \approx 3 \times 10^{-10} \mathrm{~m}$ through $b_{\|} \approx \eta / a \approx 10^{6} \mathrm{~J} \cdot \mathrm{s} / \mathrm{m}^{4}$. As mentioned before, the friction coefficient $b_{\perp}$ is the inverse of the membrane permeation coefficient; it is known to be $b_{\perp} \approx 10^{12} \mathrm{~J} \cdot \mathrm{s} / \mathrm{m}^{4}$ [13], which is far larger than $b_{\|}$. With these numerical values, the three crossover wavenumbers can be roughly estimated as $q_{1} \approx 10^{6} \mathrm{~m}^{-1}$, $q_{2} \approx 10^{14} \mathrm{~m}^{-1}$, and $q_{3} \approx 10^{9} \mathrm{~m}^{-1}$. Therefore, we recognize that $q_{2}$ is beyond the appropriate range for the present theory and that the scaling behavior $\gamma_{1} \sim q^{4}$ for $q \gg q_{2}$ should not be observable.

As for the scale dependent viscosities in Eq. (20), we expect essentially $\eta_{\perp} \approx \eta$ because $b_{\perp}$ is very large. Besides, $\eta_{\|}$is generally dependent on $q$ and becomes $\eta_{\|} \approx b_{\|} /(2 q)$ when $q \gg q^{*}$ where $q^{*}=b_{\|} /(2 \eta) \approx 10^{8} \mathrm{~m}^{-1}$ for the above parameter values. Hence, for $q^{*} \ll q \ll q_{3}$, the monolayer friction coefficient is renormalized as $b \rightarrow$ $b+b_{\|}$. However, since $b_{\|} / b \approx 10^{-3}$ for the above typical parameter values, the modification of $b$ due to the partial slip boundary condition may not be observable. We also

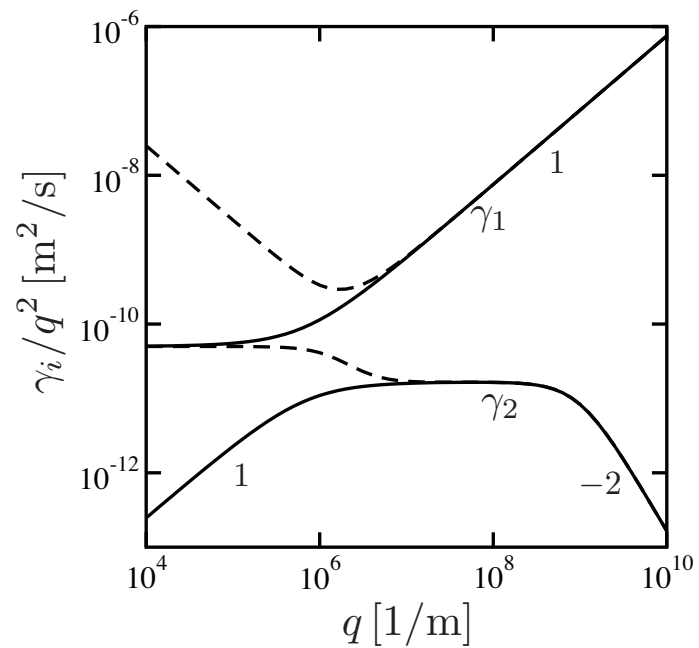

FIG. 3. The relaxation rates $\gamma_{1}$ and $\gamma_{2}$ as a function of the wavenumber $q$. The solid lines show the same data as in Fig. 2 The dashed line corresponds to the same parameters except for a finite membrane tension $\sigma=10^{-6} \mathrm{~J} / \mathrm{m}^{2}$. The latter affects only the small $q$ behaviors. Although the partial slip boundary conditions have been taken into account they also make no visible difference here.

note that $q^{*}$ is already close to $q_{3}$. From these results, one can conclude the effects of partial slip boundary conditions do not show up in the relaxation dynamics of a compressible bilayer membrane. In other words, is it justified to neglect both permeation and parallel slip at the membrane surface for ordinary lipid membranes.

Using the above parameter values, we numerically calculate the two relaxation rates $\gamma_{1}$ and $\gamma_{2}$ as shown in Fig. 2 (solid lines). Although the partial slip boundary conditions are included here, the result is essentially the same as that by Seifert and Langer [4] because of the above mentioned reasons. The wavenumber dependencies of the relaxation rates are in accordance with the asymptotic behaviors in Eqs. (21) and (22), although we do not see the dependence $\gamma_{1} \sim q^{4}$ for $q \gg q_{2}$ since $q_{2}$ is too large. Just for comparison, we have also plotted in Fig. 2 the relaxation rates for $b_{\perp} \approx 10^{6} \mathrm{~J} \cdot \mathrm{s} / \mathrm{m}^{4}$ (dashed line) which corresponds to a very permeable membrane. Here $\gamma_{1}$ increases as $\gamma_{1} \sim q^{4}$ for $q \gg 10^{8} \mathrm{~m}^{-1}$. In Fig. B we show the effect of a finite membrane tension of the relaxation rates, as presented by the dashed line.

It is instructive to compare here the different dissipation mechanisms introduced in the Rayleighian. The dissipation due to the inter-monolayer friction is given by Eq. (4). In Fourier space, using the mass conservation law, it can be rewritten as

$$
\mathcal{P}_{i}=\int \frac{d^{2} q}{(2 \pi)^{2}} \frac{b}{2 q^{2}}|\dot{\rho}(\mathbf{q})|^{2},
$$

Similarly, one can Fourier transform the dissipation $\mathcal{P}_{\|}^{ \pm}$ [see Eq. [6])] due to the friction between the monolayer 
and the bulk fluid:

$$
\mathcal{P}_{\|}^{ \pm}=\int \frac{d^{2} q}{(2 \pi)^{2}} \frac{b_{\|}}{q^{2}}\left(\frac{\eta q}{b_{\|}+2 \eta q}\right)^{2}|\dot{\rho}(\mathbf{q})|^{2} .
$$

Looking at the $q$-dependent coefficient of $|\dot{\rho}(q)|^{2}$ in the integrand, we see that it decays as $q^{-2}$ in Eq. (23), whereas in Eq. (24), it is independent of $q$ when $q \ll q^{*}$, while it also decays as $q^{-2}$ for $q \gg q^{*}$. Hence $\mathcal{P}_{i}$ always dominates for $q \ll q^{*}$, whereas the sum $b+b_{\|}$contributes to the dissipation for $q \gg q^{*}$. This is consistent with the previous argument on the renormalized friction coefficient, but we note again that $b_{\|}$is typically much smaller than $b$ for ordinary membranes.

In Figs. 2 and 3 we have plotted up to $q=10^{10} \mathrm{~m}^{-1}$. Although directly detecting molecular scale dynamics may not be so easy, we note that a long-wavelength deformation can excite a collection of modes with much shorter wavelengths, e.g., when a bilayer membrane is coupled with a cytoskeleton [10]. This is because the lattice structure of a cytoskeleton breaks lateral continuous translational symmetry and couples Fourier modes with different wave vectors.

Some years ago, Müller and Müller-Plathe investigated shear viscosity of a lipid bilayer system by using reverse non-equilibrium molecular dynamics simula- tions [14]. They showed that water molecules are less mobile near the lipid headgroups than in the bulk water, and the local viscosity of water close to the headgroup interface is several times larger than the bulk water viscosity. This means that the parallel friction coefficient $b_{\|} \approx \eta / a$ can be even larger than our estimate (assuming $a$ is the same) because we have used the bulk water viscosity value for $\eta$. In our theory, the increase of $b_{\|}$leads to the increase of the crossover wavelength $q^{*}$.

In conclusion, we have studied the effect on membrane dynamics of partial slip boundary conditions at the monolayers-solvent interface. We found that a new regime may appear in the spectrum of the relaxation rates and that the new friction coefficients associated with the partial slip boundary conditions renormalize the solvent viscosity. For ordinary lipid bilayer membranes, however, these effect should not be detectable and it is plainly justified to ignore them. It is nonetheless possible that exotic membranes may someday display the new regimes that we have calculated.

S.K. and R.O. acknowledge support by Grant-in-Aid for Scientific Research on Innovative Areas "Fluctuation and Structure" (Grant No. 25103010) from the Ministry of Education, Culture, Sports, Science, and Technology (MEXT) of Japan, and by Grant-in-Aid for Scientific Research (C) (Grant No. 15K05250) from the Japan Society for the Promotion of Science (JSPS).
[1] B. Alberts, A. Johnson, P. Walter, J. Lewis, M. Raff, Molecular Biology of the Cell (Garland Science, New York, 2008).

[2] F. Brochard and J. F. Lennon, J. Phys. (Paris) 36, 1035 (1975).

[3] R. Merkel, E. Sackmann, and E. Evans, J. Phys. (France) 50, 1535 (1989).

[4] U. Seifert and S. A. Langer, Europhys. Lett. 23, 71 (1993).

[5] M. Mell, L. H. Moleiro, Y. Hertle, I. López-Montero, F. J. Cao, P. Fouquet, T. Hellweg, and F. Monroy, Chem. Phys. Lipids 185, 61 (2015).

[6] R. Okamoto, Y. Kanemori, S. Komura, and J.-B. Fournier, Eur. Phys. J. E 39, 52 (2016).

[7] L. Miao, M. A. Lomholt, and J. Kleis, Eur. Phys. J. E 9,
$143(2002)$.

[8] T. V. Sachin Krishnan, R. Okamoto, and S. Komura, Phys. Rev. E 94, 062414 (2016).

[9] T. V. Sachin Krishnan, R. Okamoto, and S. Komura, to be published in J. Phys.: Condens. Matter.

[10] R. Okamoto, S. Komura, and J.-B. Fournier, Phys. Rev. E 96, 012416 (2017).

[11] M. Doi, Soft Matter Physics (Oxford University, Oxford, 2013).

[12] J.-B. Fournier, Int. J. Nonlinear Mech. 75, 67 (2015).

[13] J.-B. Manneville, P. Bassereau, S. Ramaswamy, and J. Prost, Phys. Rev. E 64, 021908 (2001).

[14] T. J. Müller and F. Müller-Plathe, ChemPhysChem 10, 2305 (2009). 